\title{
How does scapula motion change after reverse total shoulder arthroplasty? - a preliminary report
}

\author{
Myung-Sun Kim¹, Keun-Young Lim², Dong-Hyun Lee ${ }^{1}$, David Kovacevic ${ }^{3}$ and Nam-Young Cho ${ }^{1 *}$
}

\begin{abstract}
Background: Arm elevation is composed of glenohumeral and scapulothoracic motion. Many reports have addressed changes of scapular position across a spectrum of shoulder disease. However, no study has examined changes in scapular position after reverse total shoulder arthroplasty (RTSA). The purpose of this study was to evaluate the changes in scapular position after RTSA compared to patients' contralateral, nonoperated shoulder.

Methods: Seven patients that underwent RTSA for cuff tear arthropathy from July 2007 to October 2008 were enrolled. The distance between the long axis of the thoracic spine and the inferior pole of the scapula (lateralization of the scapula) was measured on shoulder A-P radiographs at 0 degrees (the neutral position) and at $30,60,90$, and 120 degrees of shoulder abduction. In addition, the angle between the long axis of the thoracic spine and medial border of the scapula was measured and compared with the patients' contralateral shoulder.

Results: Scapulohumeral rhythm was 2.4:1 on the operated shoulder and 4.1:1 on the nonoperated, contralateral shoulder at 120 degrees of abduction. The distance between the line of the interspinous process of upper thoracic vertebra and the inferior pole of the scapula showed a negative slope at 0 to 30 degrees abduction on the operated side, but beyond 30 degrees of abduction, this distance showed a more sudden increase than in the contralateral shoulder. The angle between the vertical vertebral line and the scapular medial border also showed greater increase beyond 30 degrees abduction on the operated limb.
\end{abstract}

Conclusions: The pattern of scapular position after RTSA, was found to differ from that of the contralateral shoulder, and showed a more scapular upward rotation.

Keywords: Scapular position, Scapulohumeral rhythm, Reverse Total Shoulder Arthroplasty (RTSA)

\section{Background}

Arm elevation is composed of glenohumeral (GH) and scapulothoracic (ST) joint motion. This was first called scapulohumeral rhythm (SHR) by Codman [1] and the normal ratio was reported by Inman et al. [2] as 2:1 (Figure 1). The motion of the scapula is affected by $\mathrm{GH}$ joint motion, and according to Inman, the setting phase is in 0 to 60 degrees of abduction, where the scapula is stabilized. In the setting phase, according to Yano et al. [3], scapular motion is reduced, and beyond 60 degrees, scapulohumeral rhythm is decreased, which means more scapular motion.

Several reports have addressed changes in scapular position in many shoulder diseases, such as, impingement,

\footnotetext{
* Correspondence: myelysium@paran.com

'Department of Orthopaedic Surgery, Chonnam National University College of Medicine, 671, Jebong-Ro, Dong-Gu, Gwangju 501-757, South Korea Full list of author information is available at the end of the article
}

rotator cuff tear, instability, and frozen shoulder [4-9] while investigation of scapulohumeral rhythm after total shoulder arthroplasty has been performed as well [10]. However, the reverse total shoulder arthroplasty, which is indicated when patients have rotator cuff tear arthropathy or irreparable massive rotator cuff tear with poor motion, biomechanically differs from the native shoulder and anatomic shoulder arthroplasty. Reverse total shoulder arthroplasty medializes the center of rotation, distalizes the humerus, and elongates the deltoid. The lever arm of the deltoid muscle is lengthened so that for any given angular displacement of the humerus, shortening of the deltoid is greater than for total shoulder arthroplasty. Furthermore, no study has previously reported changes in scapular position after reverse total shoulder arthroplasty.

Thus, the purpose of this study was to evaluate the changes in scapular position after reverse total shoulder

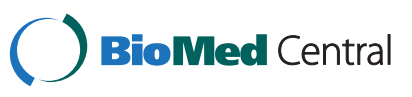




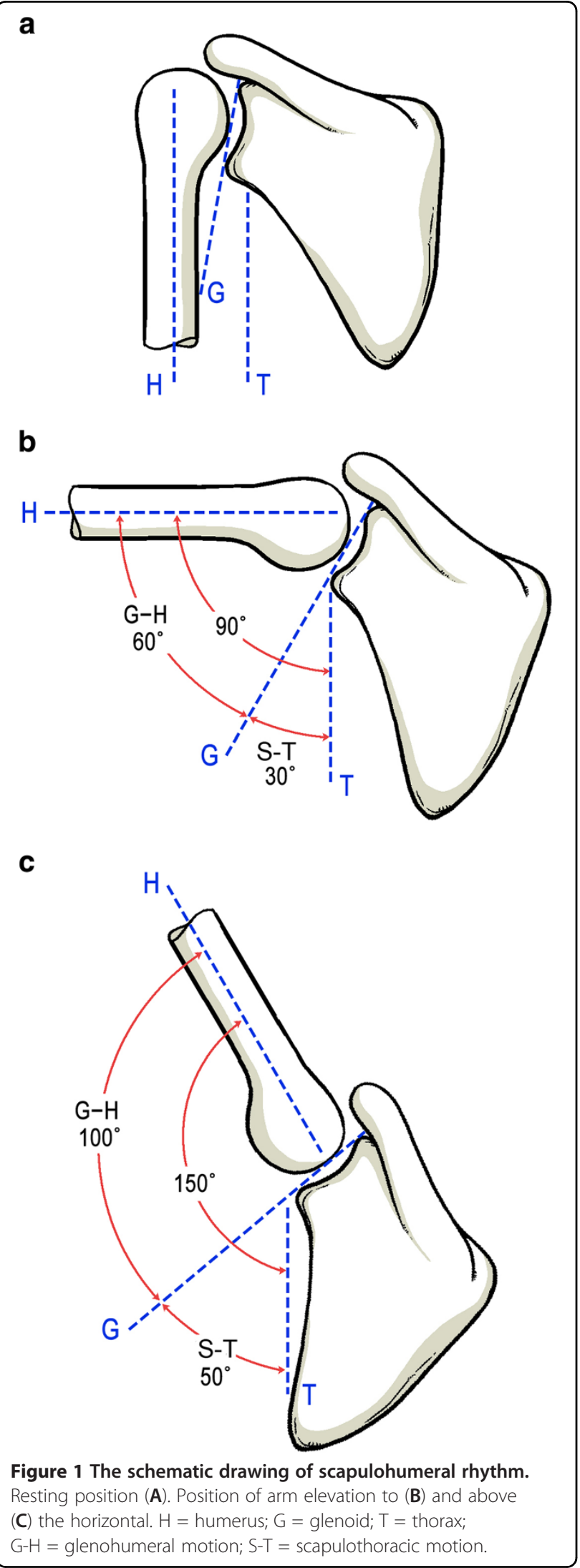

arthroplasty compared to the nonoperated, contralateral shoulder during abduction in the scapular plane.

\section{Methods}

This study was approved by our institutional review board, and informed consent was obtained from all participants. We reviewed seven primary reverse total shoulder arthroplasties conducted in seven patients between July 2007 and October 2008. The study cohort consisted of two men and five women of mean age 67.4 years (range, 63 to 77 years) at surgery. We included the patient who did not have a history of shoulder disease in the contralateral side, and all had normal active arm elevation and abduction, and active abduction over 120 degrees in operated sides at their follow ups (mean 22.3 months postoperatively). In these seven shoulders, five shoulders had pseudoparalysis with cuff tear arthropathy (cuff tear arthropathy with less than 90 degrees of active forward elevation without actual neurologic deficit) [11], one shoulder had osteoarthritis of the glenohumeral joint and a massive rotator cuff tear, and one shoulder had humeral head osteonecrosis.

All patients were implanted with the Aequalis Reverse Shoulder Prosthesis (Tornier, Edina, MN) with a $6.5 \mathrm{~mm}$ stem, a $36 \mathrm{~mm}$ glenoid hemisphere, and a $25 \mathrm{~mm}$ glenoid baseplate. The component sizes were chosen because they were the most commonly used sizes of the Aequalis Reverse Shoulder system. All patients were placed in the beach chair position and the deltopectoral approach was used to gain access to the shoulder joint. In those cases where the inferior third of the subscapularis tendon was intact, it was released from the lesser tuberosity and preserved for reinsertion at the end of the procedure with transosseous sutures. Using the forearm as a reference, the humeral head osteotomy was performed with a jig to achieve 20 degrees retroversion and 150 degrees inclination. Next, sequentially larger reamers were used to open and prepare the humeral canal. The trial prosthesis was kept in place to protect the proximal humerus during glenoid preparation. The guide wire for the glenoid reamer was positioned so that the glenoid baseplate could be placed as low as possible such that it was flush with the inferior glenoid rim. Reaming was performed manually and cranial and caudal divergently-directed locking screws as well as anterior and posterior nonlocking screws were used to secure the glenoid baseplate to provide primary stability. An appropriately sized glenoid hemisphere was then mounted on the baseplate. Polyethylene insert thickness for the humeral component was chosen based on soft-tissue tension during trial reduction. After surgery, patients were placed in an abduction sling for 6 weeks. The initiation of passive range of motion with low intensity supervised physical therapy was started 4 weeks after 
surgery, and progressed from active assisted range of motion to full active range of motion. Passive external rotation was initially avoided to protect the healing subscapularis tendon repair [12].

We used two clinical outcome reporting tools to measure pain and function after surgery compared to patients' preoperative shoulder score. The American Shoulder and Elbow Surgeons (ASES) Shoulder score evaluates pain and activities of daily living reported by the patient. The Korean Shoulder Score (KSS) evaluates function, pain, patient satisfaction, active range of motion in two planes (i.e., forward elevation, internal / external rotation), strength, and endurance. In addition, we measured scapular position at last follow-up by obtaining a standing AP view of the shoulder in the scapular plane at 0 , 30, 60, 90 and 120 degrees of abduction. To evaluate scapular position, the distance from the interspinous process of the thoracic vertebra to the inferior angle of the scapula was measured and reported in millimeters (Figure 2A). Scapular tilt was measured as the angle formed between a vertical line bisecting the vertebral body and an oblique line drawn from the superior to inferior angle (i.e., scapular medial border) (Figure 2B).
The scapular position and tilt was determined for each operative shoulder and then compared with that of the contralateral shoulder by consensus agreement amongst the authors (MSK, KYL, DHL, and NYC).

\section{Results}

Mean active forward elevation after surgery was 138.6 degrees, and abduction was 131.2 degrees. The mean visual analog scale score improved from 8.4 preoperatively to 0.8 points postoperatively. The mean ASES shoulder score improved from 30.0 to 83.3 points, and KSS score improved from 37.4 to 76.5 points.

The distances between the interspinous process of the upper thoracic vertebra and the inferior pole of the scapula on operated shoulders were $85.2 \mathrm{~mm}$ at 0 degrees, $73.1 \mathrm{~mm}$ at 30 degrees, $91.4 \mathrm{~mm}$ at 60 degrees, $110.1 \mathrm{~mm}$ at 90 degrees and $138.5 \mathrm{~mm}$ at 120 degrees of abduction. In the contralateral shoulders, these distances between the vertical vertebral line and the inferior pole of the scapula were 81.4, 90.5, 104.4, 111.8 , and $131.1 \mathrm{~mm}$ at $0,30,60,90$ and 120 degrees of abduction, respectively (Figure 3 ). The difference at each interval of motion was $-12.1,18.3,18.7$, and $28.4 \mathrm{~mm}$ for
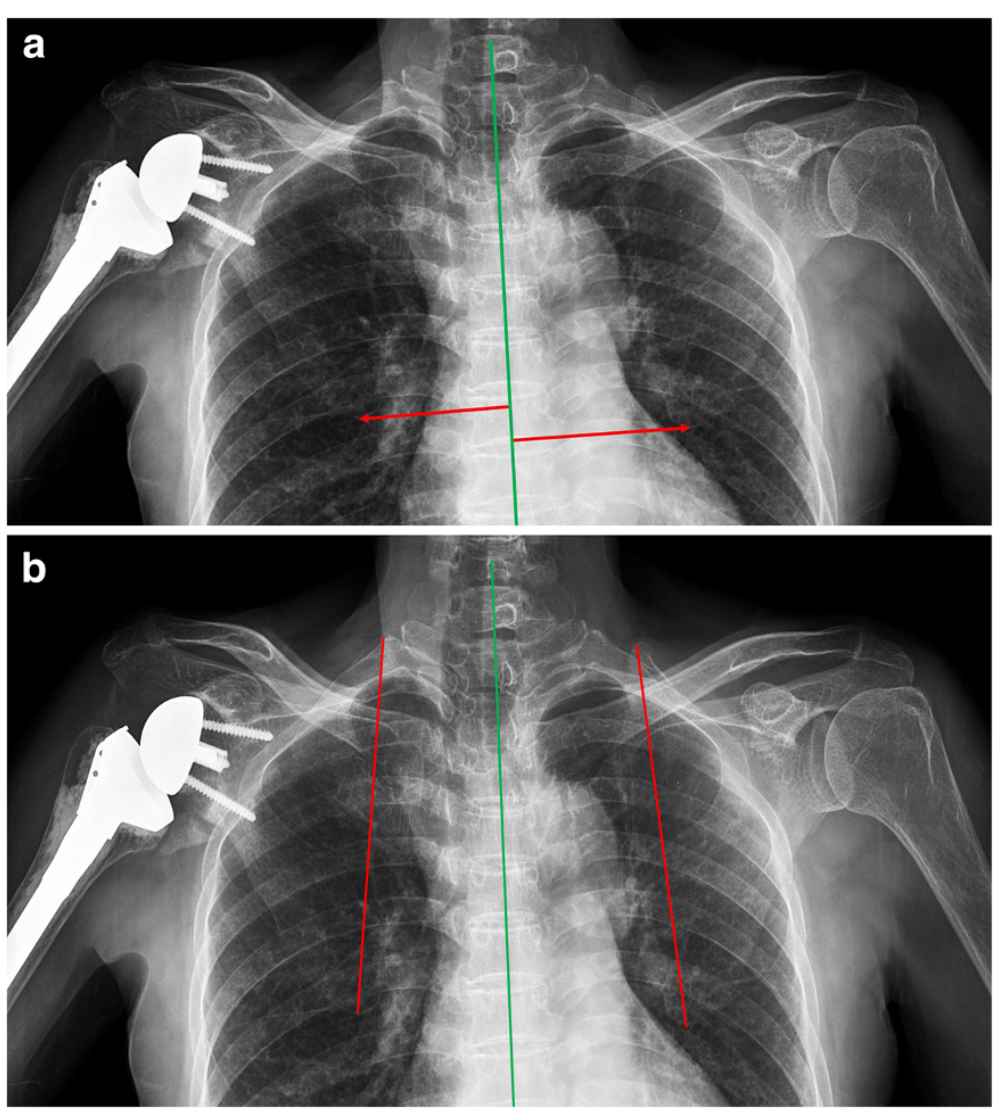

Figure 2 The measurement method of the distance from the interspinous process of the thoracic vertebra to the inferior angle of the scapula was measured and reported in millimeters (A). Scapular tilt was measured as the angle formed between a vertical line bisecting the vertebral body and an oblique line drawn from the superior to inferior angle (B). 


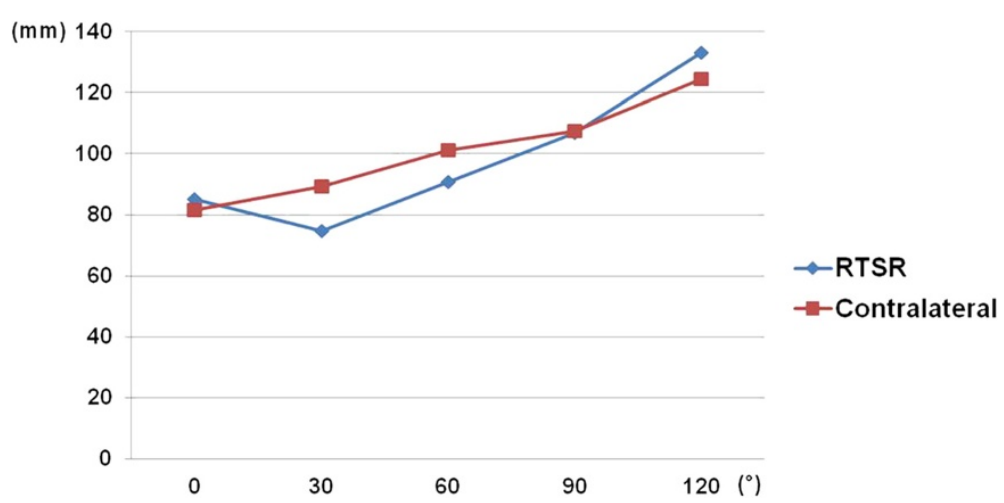

Figure 3 The distance from the interspinous process of the thoracic vertebra to the inferior angle of the scapula.

the operated shoulders, and 9.1, 13.9, 7.4, and $19.3 \mathrm{~mm}$ for the contralateral shoulders. From 0 to 30 degrees in the abduction arc of motion for the operated shoulders, the slope was negative. However, beyond 30 degrees of abduction, slope of distance showed more sudden increase of distance than the contralateral shoulder (Table 1).

The angles between the vertical vertebral line and the scapular medial border on operated shoulders were 5.5, 6.3, 20.1, 26.4, and 39.0 degrees at $0,30,60,90$, and 120 degrees of abduction, respectively, and on the contralateral shoulders, these angles were 7.7, 9.7, 16.2, 22.0, and 30.0 degrees, respectively (Figure 4 ). The difference at each interval of motion was $0.8,13.8,6.3$, and 12.6 degrees on the operative limb, and 2, 6.5, 5.8, and 8 degrees on the contralateral limb. The slope of the angle also showed more increase beyond 30 degrees abduction after surgery compared to the nonoperative limb (Table 1).

Mean scapulohumeral rhythm was $2.4: 1$ on operated sides and 4.1:1 on contralateral sides at 120 degrees of abduction, showing less glenohumeral motion and more

\begin{tabular}{|c|c|c|c|c|}
\hline \multirow[t]{2}{*}{ Interval } & \multicolumn{2}{|c|}{ Distance $^{*}(\mathrm{~mm})$} & \multicolumn{2}{|c|}{ Angle $^{\dagger}\left({ }^{\circ}\right)$} \\
\hline & RTSA & Normal & RTSA & Normal \\
\hline $0^{\circ}-30^{\circ}$ & -12.1 & 9.1 & 0.8 & 2.0 \\
\hline $30^{\circ}-60^{\circ}$ & 18.3 & 13.9 & 13.8 & 6.5 \\
\hline $60^{\circ}-90^{\circ}$ & 18.7 & 7.4 & 6.3 & 5.8 \\
\hline $90^{\circ}-120^{\circ}$ & 28.4 & 19.3 & 12.6 & 8.0 \\
\hline
\end{tabular}

RTSA = reverse total shoulder arthroplasty; Distance* $=$ The distance between line of inter-spinous process of the upper thoracic vertebra and inferior pole of scapula; Angle $^{\dagger}=$ The angle between line of inter-spinous process of upper thoracic vertebra and scapular medial border. scapular motion in operated shoulders compared to nonoperated, contralateral shoulders (Table 2).

\section{Discussion}

Knowledge about scapular movement under the influence of glenohumeral motion aids the understanding of the mechanics and pathology of the shoulder joint. Cathcart [13] was the first to recognize the contribution made by the scapulothoracic joint to normal shoulder complex kinematics. Codman [1] termed this synchronous motion as scapulohumeral rhythm (SHR). Inman et al. [2] reported a SHR of 2:1 in healthy subjects. Since, much research on shoulder kinematics has been directed toward the study of SHR $[2,14,15]$. Braman et al. [10] reported that shoulder motion differed between patients with advanced glenohumeral osteoarthritis and healthy individuals, and that total shoulder arthroplasty, restored SHRs to normal values.

In the present study, SHR was 2.4:1 on operated shoulders and 4.1:1 on contralateral shoulders at 120 degrees of abduction. This means that there was less glenohumeral motion and more scapular motion in operated shoulders compared to nonoperated, contralateral shoulders. We consider this postoperative change a useful adaptation of scapular mechanics to maintain the tension of the deltoid muscle to generate the forces necessary for shoulder motion, as was mentioned by Mell et al. [7].

In particular, because the function of deltoid muscle is important and the rotator cuff has no function in patients with an implanted reverse total shoulder system, this scapular motion represents a highly meaningful change. However, we carefully propose the hypothesis that that increased scapular motion after RTSR might be able to lead more stretching and fatigue of periscapular muscles at long term follow up after surgery. The specific rehabilitation programs might be needed to be designed to prevent periscapular muscle fatigue after RTSA.

In the present study, during 0 to 30 degrees of abduction, distance between the vertical vertebral line and 


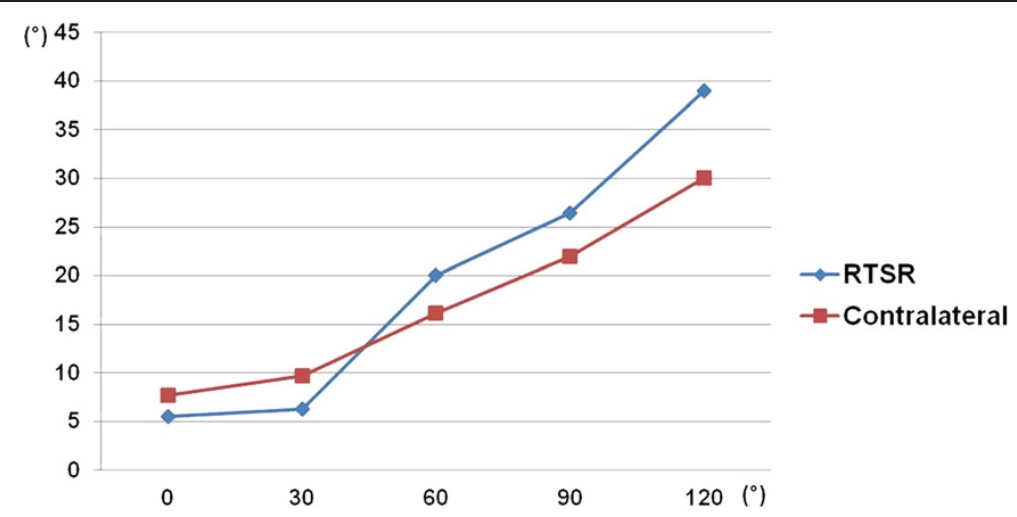

Figure 4 The angle formed between a vertical line bisecting the vertebral body and an oblique line drawn from the superior to inferior angle.

inferior pole of the scapula was decreased on operated shoulders, which means downward rotation of the scapula on initial abduction; described as motion of the glenohumeral type by Yano et al. [3]. Furthermore, the angle between the vertical vertebral line and scapular medial border increased less than in contralateral shoulders on initial abduction. However, beyond 30 degrees, changes in distance and angle were higher than in contralateral shoulders. Therefore, more scapular upward rotation occurred during middle and late abduction.

Yano et al. [3] proposed two types of upward rotation during the initial phase of elevation. For the glenohumeral type (much glenohumeral motion), the scapula slightly rotates downward and then progresses upward, and for the scapulothoracic type (much scapular motion), the scapula directly rotates upward. Inman et al. [2] used the term 'the setting phase' to describe the early phase of shoulder motion during the first 60 degrees of abduction, indicating preparatory stabilization of the scapula to permit controlled humeral motion. Yano et al. [16] reported that SHR was generally greatest (less scapular motion) during the setting phase and that it then decreased beyond 60 degrees of abduction (more scapular motion). Furthermore, the SHR increased again below 60 degrees of abduction. They mentioned that muscular stabilization of the

Table 2 The degree of glenohumeral and scapulohumeral rhythm on operated and contralateral shoulder at $30^{\circ}$, $60^{\circ}, 90^{\circ}, 120^{\circ}$ of abduction

\begin{tabular}{rcccr}
\hline Abduction & RTSA & \multicolumn{3}{c}{ Normal } \\
\cline { 2 - 5 } & G-H & S-T & G-H & S-T \\
\hline $30^{\circ}$ & $23.8^{\circ}$ & $0.7^{\circ}$ & $20.3^{\circ}$ & $2.0^{\circ}$ \\
$60^{\circ}$ & $39.9^{\circ}$ & $14.6^{\circ}$ & $43.8^{\circ}$ & $8.5^{\circ}$ \\
$90^{\circ}$ & $63.6^{\circ}$ & $20.9^{\circ}$ & $68.0^{\circ}$ & $14.3^{\circ}$ \\
$120^{\circ}$ & $81.0^{\circ}$ & $33.5^{\circ}$ & $90.0^{\circ}$ & $22.3^{\circ}$ \\
\hline
\end{tabular}

RTSA = reverse total shoulder arthroplasty; G-H = glenohumeral motion; $\mathrm{S}-\mathrm{T}=$ scapulothoracic motion. scapula increases while raising the arms, and thus, that less scapular motion is seen during the setting phase.

The present study has some limitations. The most obvious of which is the small number of cases enrolled. This investigation is a pilot study of our early experience with this prosthesis. Based on our inclusion criteria and the fact that it is difficult to have patients return for follow-up, we were only able to include 7 patients at this time. In addition, we only considered upward rotation of the scapula, and not the other planes. Nevertheless, the study has relevance in the context of evaluating changes of scapular upward rotation after reverse total shoulder arthroplasty. Because it is the main movement of the scapula, upward rotation is frequently addressed during treatment and research [17-19]. This preliminary work is a proof of concept study. Future work will include more patients and may be able to use of CT to measure scapular position.

\section{Conclusions}

The pattern of scapular position after RTSA was found to differ between operated shoulders and nonoperated, contralateral shoulders. In particular, there was more upward rotation after surgery.

\section{Competing interests}

The authors declare that they have no competing interests.

\section{Authors'contributions}

MSK designed this study, reviewed the literature and drafted the manuscript. KBL and KYL gave substantial intellectual ideas in drafting the manuscript. NYC participated in the acquisition of data of cases and gave substantial contributetion to interpretation of the literature review. DK gave substantial contributions to revision of manuscript, intellectual ideas, and editorial direction. All authors read and approved the final manuscript.

\section{Acknowledgements}

No funding was received for this research. None of the authors have any conflict of interest or disclosures to report in relation to this work. 


\section{Author details}

1 Department of Orthopaedic Surgery, Chonnam National University College of Medicine, 671, Jebong-Ro, Dong-Gu, Gwangju 501-757, South Korea. 2Department of Orthopaedic Surgery, Gwangju Hospital, 565-1, Duam-Dong Buk-Gu, Gwangju 500-100, South Korea. ${ }^{3}$ Department of Orthopaedic Surgery, Cleveland Clinic Foundation, 9500 Euclid Ave., A-41, Cleveland, OH 44195, USA.

Received: 14 December 2011 Accepted: 11 October 2012

Published: 29 October 2012

\section{References}

1. Codman EA: The shoulder. Boston: Thomas Todd Company; 1934:32-64.

2. Inman VT, Saunders JB, Abbott LC: Observations of the function of the shoulder joint. 1944. Clin Orthop Relat Res 1996, 330(9):3-12.

3. Yano Y, Hamada J, Tamai K, Yoshizaki K, Sahara R, Fujiwara T, Nohara Y: Different scapular kinematics in healthy subjects during arm elevation and lowering: glenohumeral and scapulothoracic patterns. J Shoulder Elbow Surg 2010, 19(2):209-215.

4. Kibler WB, Uhl TL, Maddux JW, Brooks PV, Zeller B, McMullen J: Qualitative clinical evaluation of scapular dysfunction: a reliability study. J Shoulder Elbow Surg 2002, 11(6):550-556.

5. Ludewig PM, Cook TM, Nawoczenski DA: Three-dimensional scapular orientation and muscle activity at selected positions of humeral elevation. J Orthop Sports Phys Ther 1996, 24(2):57-65.

6. Lukasiewicz AC, McClure P, Michener L, Pratt N, Sennett B: Comparison of 3-dimensional scapular position and orientation between subjects with and without shoulder impingement. J Orthop Sports Phys Ther 1999, 29(10):574-583. discussion 584-6.

7. Mell AG, LaScalza S, Guffey P, Ray J, Maciejewski M, Carpenter JE, Hughes RE: Effect of rotator cuff pathology on shoulder rhythm. J Shoulder Elbow Surg 2005, 14(1 Suppl S):58S-64S.

8. Rundquist PJ, Anderson DD, Guanche CA, Ludewig PM: Shoulder kinematics in subjects with frozen shoulder. Arch Phys Med Rehabil 2003, 84(10):1473-1479.

9. Warner JJ, Micheli LJ, Arslanian LE, Kennedy J, Kennedy R: Scapulothoracic motion in normal shoulders and shoulders with glenohumeral instability and impingement syndrome. A study using Moire topographic analysis. Clin Orthop Relat Res 1992, 285(12):191-199.

10. Braman JP, Thomas BM, Laprade RF, Phadke V, Ludewig PM: Threedimensional in vivo kinematics of an osteoarthritic shoulder before and after total shoulder arthroplasty. Knee Surg Sports Traumatol Arthrosc 2010, 18(12):1774-1778.

11. Boileau P, Chuinard C, Roussanne Y, Bicknell RT, Rochet N, Trojani C: Reverse shoulder arthroplasty combined with a modified latissimus dorsi and teres major tendon transfer for shoulder pseudoparalysis associated with dropping arm. Clin Orthop Relat Res 2008, 466(3):584-593.

12. Nolan BM, Ankerson E, Wiater JM: Reverse total shoulder arthroplasty improves function in cuff tear arthropathy. Clin Orthop Relat Res 2011, 469(9):2476-2482

13. Cathcart CW: Movements of the Shoulder Girdle involved in those of the Arm on the Trunk. J Anat Physiol 1884, 18(Pt 2):211-218.

14. Bagg SD, Forrest WJ: A biomechanical analysis of scapular rotation during arm abduction in the scapular plane. Am J Phys Med Rehabil 1988, 67(6):238-245

15. Poppen NK, Walker PS: Normal and abnormal motion of the shoulder. Surg Forum 1975, 26:519.

16. Yano Y, Tamai K, Nohara Y, Yoshizaki K, Hamada J: Do healthy subjects elevate both shoulders in the same manner? [in Japanese]. Shoulder Joint 2008, 80(3):241-244.

17. Ludewig PM, Cook TM: Alterations in shoulder kinematics and associated muscle activity in people with symptoms of shoulder impingement. Phys Ther 2000, 80(3):276-291.
18. McQuade KJ, Dawson J, Smidt GL: Scapulothoracic muscle fatigue associated with alterations in scapulohumeral rhythm kinematics during maximum resistive shoulder elevation. J Orthop Sports Phys Ther 1998, 28(2):74-80

19. Ludewig PM, Reynolds JF: The association of scapular kinematics and glenohumeral joint pathologies. J Orthop Sports Phys Ther 2009, 39(x):90-104.

doi:10.1186/1471-2474-13-210

Cite this article as: Kim et al: How does scapula motion change after reverse total shoulder arthroplasty? - a preliminary report. $B M C$ Musculoskeletal Disorders 2012 13:210

\section{Submit your next manuscript to BioMed Central and take full advantage of:}

- Convenient online submission

- Thorough peer review

- No space constraints or color figure charges

- Immediate publication on acceptance

- Inclusion in PubMed, CAS, Scopus and Google Scholar

- Research which is freely available for redistribution

Submit your manuscript at www.biomedcentral.com/submit
C Biomed Central 